\title{
Administration of Intracoronary Streptokinase During Primary Percutaneous Coronary Intervention for Anterior Wall Myocardial Infarction with Definite Coronary Thrombosis
}

\author{
Haytham Fathalla Emara ${ }^{1}$, Wael Mahmoud EI Kilany ${ }^{2}$, Tarek Mounir Zaki ${ }^{2}$, \\ Ahmed Mohamed El-Missiri ${ }^{2, *}$ \\ ${ }^{1}$ Cardiology Department, National Heart Institute, Cairo, Egypt \\ ${ }^{2}$ Cardiology Department, Ain Shams University, Cairo, Egypt
}

Email address:

amissiri@med.asu.edu.eg (A. M. El-Missiri)

${ }^{*}$ Corresponding author

\section{To cite this article:}

Haytham Fathalla Emara, Wael Mahmoud El Kilany, Tarek Mounir Zaki, Ahmed Mohamed El-Missiri. Administration of Intracoronary Streptokinase During Primary Percutaneous Coronary Intervention for Anterior Wall Myocardial Infarction with Definite Coronary Thrombosis. Cardiology and Cardiovascular Research. Vol. 4, No. 1, 2020, pp. 11-16. doi: 10.11648/j.ccr.20200401.13

Received: February 2, 2020; Accepted: February 14, 2020; Published: February 24, 2020

\begin{abstract}
Background: The presence of intracoronary heavy thrombus burden during primary percutaneous coronary intervention (PCI) plays increases the incidence of occurrence no-reflow phenomenon. Intracoronary thrombolytic therapy during primary PCI may improve microvascular perfusion. The aim of this study was to assess the effect of using 250,000 $\mathrm{U}$ of intracoronary streptokinase during primary PCI in patients presenting with an acute anterior wall ST segment elevation myocardial infarction (STEMI) with a definite thrombus in the left anterior descending coronary artery (LAD) on clinical and angiographic outcomes. Methods: Prospective cohort study conducted on 100 patients managed by primary PCI within 12 hours of symptom onset. Patients were divided into a study group $(n=50)$ that received intracoronary streptokinase during primary PCI, and a control group $(\mathrm{n}=50)$ that received no additional therapy. Post-procedural TIMI flow grade, myocardial blush grade (MBG), and corrected TIMI frame count were assessed. Admission and peak CK-MB and percentage of ST segment resolution were recorded. At 6-months follow-up, assessment for major adverse cardiovascular events (MACE) was performed. Results: There were no differences between both groups regarding baseline clinical characteristics, time to reperfusion, and risk factors for the development of coronary artery disease. Peak CK-MB was significantly higher in the control group ( $\mathrm{p}=0.004)$. In the study group, a larger proportion of patients had TIMI 3 flow at the end of the procedure 42 $(84 \%)$ vs $29(58 \%)-p=0.026$, and a larger proportion had MBG 2 and 3, $23(46 \%)$ vs $17(34 \%)$ and $24(48 \%)$ vs $14(28 \%)$, respectively $-\mathrm{p}=0.001$. Corrected TIMI frame count at the end of the procedure was significantly smaller in the study group $24.2 \pm 4.97$ vs $31.28 \pm 6.7$ frames $(\mathrm{p}<0.0001)$. Conclusion: Administration of intracoronary streptokinase during primary PCI in patients presenting with acute anterior STEMI with definite coronary thrombosis improves coronary perfusion by improving TIMI flow grade, MBG, and shortening corrected TIMI frame count.
\end{abstract}

Keywords: Intracoronary Streptokinase, Coronary Thrombus, Myocardial Infarction, Primary Percutaneous Coronary Intervention

\section{Introduction}

Primary percutaneous coronary intervention $(\mathrm{PCI})$ is the mainstay treatment strategy for patients presenting with acute ST-segment elevation myocardial infarction (STEMI) according to current practice guidelines $[1,2]$.

Patients presenting with a significant intracoronary thrombus burden often present a challenge due to the increased incidence of occurrence of the no-reflow phenomenon. This is often associated with impaired 
myocardial blush grade and distal embolization of the coronary vasculature. No-reflow causes an increased risk of major adverse cardiovascular events (MACE) [3].

Over the years, several preventative and treatment options were applied including mechanical aspiration, embolic protection devices, rheolytic thrombectomy, ischemic preconditioning, as well as, the use of pharmacological agents such as anticoagulants, glycoprotein IIb/IIIa antagonists and vasodilators such as verapamil $[4,5]$.

The use of intracoronary fibrinolytic therapy during primary PCI was studied based on the theory that the coronary microcirculation is occluded during no-reflow by microvascular fibrin, vessel wall components, circulating blood cells, and fibrinogen. Such agents are thought to improve microvascular perfusion thus decreasing the size of the infarction and thus helping preserve left ventricular (LV) function especially in those presenting with a significant thrombus burden at the time of the myocardial infarction (MI) [6].

The aim of this study was to assess the effect of using $250,000 \mathrm{U}$ of intracoronary streptokinase during primary PCI in patients presenting with an acute anterior wall ST segment elevation myocardial infarction (STEMI) who were found to have a definite thrombus in the left anterior descending coronary artery (LAD) on clinical and angiographic outcomes.

\section{Methods}

The study protocol was approved by the institutional ethical committee. Written informed consents were provided by all participants. This was a prospective cohort study conducted in the period from July 2017 to February 2019 on 100 patients presenting to the emergency department with an acute anterior wall STEMI for the first time managed by primary PCI within 12 hours of onset of angina or angina equivalent symptoms. Patients were included only if left coronary angiography revealed a thrombus of grade 2 or more in the LAD according to the TIMI thrombus grade.

Patients were excluded from the study if they had any of the following: history of myocardial infarction (MI) or acute coronary syndrome; history of coronary artery bypass grafting or previous PCI; history of gastrointestinal bleeding; recent (less than 6 months) cerebrovascular stroke or transient ischemic attack; recent surgery (less than 3 months); known hematological disorders; an estimated glomerular filtration rate less than $60 \mathrm{ml} / \mathrm{min}$; allergy to contrast media or streptokinase; current cardiogenic shock; current systolic murmur anywhere over the precordium implying the presence of possible mechanical complications of MI.

TIMI thrombus grade was created by the TIMI group investigators and is as follows [7]: TIMI thrombus grade 0 means no angiographic characteristics of a thrombus are present; TIMI thrombus grade 1 means a possible thrombus is present (seen angiographically as a reduced contrast density, haziness, an irregular lesion contour, or a smooth convex "meniscus" at the site of total occlusion suggestive but not diagnostic of a thrombus); TIMI thrombus grade 2 means a definite thrombus whose greatest dimensions is less than or equal to half of the vessel diameter; TIMI thrombus grade 3 means a definite thrombus whose greatest dimension is more than half and less than twice the vessel diameters; TIMI thrombus grade 4 means a definite thrombus whose largest dimension is more than twice the vessel diameter; and TIMI thrombus grade 5 means total occlusion of the vessel.

ECG manifestations of STEMI were defined according to the third universal definition of MI [8] as new, or presumed new, ST segment elevation at the $\mathrm{J}$ point in two contiguous leads more than or equal to $0.1 \mathrm{mV}$ in all leads except for leads V2 and V3 where the cut-off points are more than or equal to $0.2 \mathrm{mV}$ for men 40 years or older, and more than or equal to $0.25 \mathrm{mV}$ for men younger than 40 years old, and more than or equal to $0.15 \mathrm{mV}$ for women.

\subsection{Study Protocol}

All patients who met non-angiographic inclusion criteria were given aspirin $(300 \mathrm{mg})$ and clopidogrel $(600 \mathrm{mg})$ in the emergency room. Following focused history taking and clinical examination, patients were transferred to the cardiac catheterization laboratory where primary PCI was performed by experienced, locally certified interventional cardiologists following standard protocols.

Our institution's primary PCI team is available on-site 24 hours a day, seven days a week. A dedicated cardiac catheterization laboratory for patients with acute MI is always available.

Access site (femoral or radial) was decided at the operator's discretion. Following arterial sheath insertion, the patient would be administered unfractionated heparin at a dose of 100 units per kilogram. Following left coronary angiography, only those patients who had a definite thrombus (TIMI thrombus grade of 2, 3, 4, or 5) were included in the study.

Patients were then randomly assigned to either of two treatment protocols. One group $(n=50)$ underwent conventional primary intervention (control group) while the other group $(n=50)$ was administered a 250,000 $U$ of intracoronary streptokinase (study group) during the procedure. Intracoronary streptokinase was prepared by diluting 250,000 units in $15 \mathrm{ml}$ of normal saline.

Intracoronary infusion of $200 \mu \mathrm{g}$ nitroglycerin via a guiding catheter engaged in the left coronary artery was performed immediately after achieving minimal flow (at least TIMI 1 flow) in the LAD followed by $10 \mathrm{ml}$ of the prepared streptokinase. The remaining $5 \mathrm{ml}$ of streptokinase were infused immediately after stenting and post-deployment ballooning.

Operators were allowed to administer intracoronary glycoprotein IIb/IIIa antagonists at their own discretion in both groups. However, the use of thrombus aspiration devices led to the exclusion of such patients.

\subsection{Angiographic Outcome}

At the end of the procedure we assessed TIMI flow grade, 
myocardial blush grade (MBG) and corrected TIMI frame count.

TIMI flow grade is classified as follows: TIMI 3 for full perfusion of the infarct vessel with normal flow; TIMI 2 for perfusion of the entire infarct vessel into the distal bed but with delayed flow compared with a normal artery; TIMI 1 for some penetration of contrast material beyond the point of obstruction but without perfusion of the distal coronary bed; and TIMI 0 for complete occlusion of the infarct-related artery [10].

MBG is classified as follows: $\mathrm{MBG} 3$ for normal myocardial blush or contrast density, comparable with that obtained during angiography of a contralateral or ipsilateral non-infarct-related coronary artery; MBG 2 for moderate myocardial blush or contrast density but less than that obtained during angiography of a contralateral or ipsilateral non-infarct-related coronary artery; MBG 1 for minimal myocardial blush or contrast density; and MBG 0 for no myocardial blush or contrast density [11].

TIMI frame count refers to the number of cine-frames required for contrast to reach a standardized distal landmark in the culprit coronary artery using a cine-film rate of 30 frames/second. For the LAD it is the distal-most referred to as the "pitchfork" or "moustache" which usually occurs at the apex. In case the LAD wraps around the apex, the branch closest to the apex is used. Corrected TIMI Frame Count (cTFC) adjusts for the difference in vessel length between the LAD and each of the right coronary artery and left circumflex coronary artery by dividing by 1.7 [12].

\subsection{Laboratory and Electrocardiographic Outcome}

Admission creatinine kinase (CK)-MB fraction was measured, as well as, its peak value. Degree of ST-T segment resolution within one hour following PCI was assessed compared with the admission ECG.

\subsection{Clinical Outcome}

During the patient's hospital stay and at 6 months following discharge, major adverse cardiac events (MACE) were monitored in the form of cardiac death, death, cerebrovascular accidents, recurrent MI or repeated revascularization.

\subsection{Statistics}

Data were collected, verified, revised and statistically analyzed using IBM Statistical Package for Social Sciences (SPSS) version 24.0 (SPSS Inc. 2017). All variables were examined for normality distribution. Study power was more than 80 . Descriptive statistics were presented as number and percentage for categorical variables and as mean \pm standard deviation for continuous variables. Student's t-test was used to compare means. Chi-square test was used to compare frequencies, and Fisher's exact test was used when the chisquare was inapplicable. The level of significance ( $p$ value) was set at $\leq 0.05$.

\section{Results}

\subsection{Baseline Demographic, Clinical and Laboratory Variables}

There were no differences between both groups regarding baseline clinical characteristics, time to reperfusion, and risk factors for the development of coronary artery disease (table 1). There was no difference in the number of patients with more than $70 \%$ ST-T segment resolution following PCI in both groups $(\mathrm{p}=0.22)$.

There was no difference between both groups in admission CK-MB $(\mathrm{p}=0.549)$. However, peak CK-MB was significantly higher in the control group $302(98-672)$ vs $105.5(86-209)-\mathrm{p}=0.004$ (table 1$)$.

Table 1. Demographic, clinical and laboratory variables.

\begin{tabular}{llll}
\hline Variable & Study group $(\mathbf{n}=\mathbf{5 0})$ & Control group $(\mathbf{n}=\mathbf{5 0})$ & p-value \\
\hline Age, years & $58.48 \pm 9.85$ & $57.22 \pm 11.45$ & 0.556 \\
Male gender, n (\%) & $39(78 \%)$ & $37(74 \%)$ & 0.64 \\
Current smoker, n (\%) & $31(62 \%)$ & $31(62 \%)$ & 1 \\
Hypertension, n (\%) & $27(54 \%)$ & $33(66 \%)$ & 0.221 \\
Diabetes, n (\%) & $23(46 \%)$ & $21(42 \%)$ & 0.687 \\
Dyslipidemia, n (\%) & $29(58 \%)$ & $22(44 \%)$ & 0.23 \\
Family history of premature CAD, n (\%) & $12(24 \%)$ & $13(26 \%)$ & 0.817 \\
Time to reperfusion, minutes & $302.44 \pm 188.16$ & $294.6 \pm 178.74$ & 0.831 \\
Admission CK-MB, IU/L & $39.42 \pm 10.30$ & $40.63 \pm 9.8$ & 0.549 \\
Peak CK-MB, IU/L & $105.5(86-209)$ & $302(98-672)$ & 0.004 \\
More than 70\% ST-segment resolution, n (\%) & $42(84 \%)$ & $37(74 \%)$ & 0.22 \\
\hline
\end{tabular}

Continuous variables are expressed as mean and standard deviation or median (interquartile range) whereas categorical variables are expressed as number (percentage). CAD means coronary artery disease; CK-MB means creatinine kinase MB fraction.

\subsection{Angiographic Findings and Outcome}

There was no difference between both groups regarding chosen access site with the femoral approach being the preferred by operators in both groups (table 2).
Most patients had proximal LAD stenosis or occlusion: $74 \%$ in the study group and $68 \%$ in the control group $(\mathrm{p}=$ $0.509)$. There was no difference between both groups in the distribution of different TIMI thrombus grades $(p=0.991)$ with thrombus grade 5 being predominant in both groups 
(table 2).

Balloon pre-dilation was used in nearly $40 \%$ of patients of both groups $(p=0.84)$. The use of intracoronary glycoprotein $\mathrm{IIb} / \mathrm{III}$ a antagonists was limited in both groups: $6 \%$ in the study group and $16 \%$ in the control group $(\mathrm{p}=0.11)$ (table 2).

There was no difference between both groups regarding the type of stent used (bare metal or sirolimus-eluting stent). Additionally, there was no difference between both groups regarding the mean stent length and diameter (table 2).

A larger proportion of patients had TIMI 3 flow at the end of the procedure in the study group 42 (84\%) vs 29 (58\%), while a larger proportion of those in the control group had TIMI 2, 1, and 0 flow $-\mathrm{p}=0.026$ (table 2).

A larger proportion of patients had MBG 2 and 3 at the end of the procedure in the study group $23(46 \%)$ vs 17 $(34 \%)$ and $24(48 \%)$ vs $14(28 \%)$, respectively. While a larger proportion of those in the control group had MBG 1 and $0-p=0.001$ (table 2).

Corrected TIMI frame count at the end of the procedure was significantly smaller in the study group $24.2 \pm 4.97$ vs $31.28 \pm 6.7$ frames $(\mathrm{p}<0.0001)$ (table 2$)$.

Table 2. Angiographic findings and interventional procedures.

\begin{tabular}{|c|c|c|c|}
\hline Variable & Study group $(n=50)$ & Control group $(\mathrm{n}=\mathbf{5 0})$ & p-value \\
\hline \multicolumn{4}{|l|}{ Access site } \\
\hline Right femoral artery & $48(96 \%)$ & $47(94 \%)$ & \multirow{2}{*}{1} \\
\hline Right radial artery & $2(4 \%)$ & $3(6 \%)$ & \\
\hline Proximal LAD stenosis, n (\%) & $37(74 \%)$ & $34(68 \%)$ & 0.509 \\
\hline \multicolumn{4}{|l|}{ TIMI thrombus grade on angiography } \\
\hline 2 & $9(18 \%)$ & $9(18 \%)$ & \multirow{4}{*}{0.991} \\
\hline 3 & $5(10 \%)$ & $6(12 \%)$ & \\
\hline 4 & $2(4 \%)$ & $2(4 \%)$ & \\
\hline 5 & $34(64 \%)$ & $33(66 \%)$ & \\
\hline Intracoronary GP IIb/IIa use, n (\%) & $3(6 \%)$ & $8(16 \%)$ & 0.11 \\
\hline Balloon pre-dilatation, n (\%) & $21(42 \%)$ & $22(44 \%)$ & 0.84 \\
\hline \multicolumn{4}{|l|}{ Stent type } \\
\hline Bare metal stent, n (\%) & $26(52 \%)$ & $22(44 \%)$ & 0.842 \\
\hline Sirolimus eluting stent, n (\%) & $24(48 \%)$ & $28(56 \%)$ & 0.545 \\
\hline Stent diameter, mm & $3.14 \pm 0.34$ & $3.09 \pm 0.32$ & 0.449 \\
\hline \multicolumn{4}{|l|}{ Postprocedural TIMI flow } \\
\hline TIMI 0 & $0(0 \%)$ & $1(2 \%)$ & \multirow{4}{*}{0.026} \\
\hline TIMI 1 & $1(2 \%)$ & $6(12 \%)$ & \\
\hline TIMI 2 & $7(14 \%)$ & $14(28 \%)$ & \\
\hline TIMI 3 & $42(84 \%)$ & $29(58 \%)$ & \\
\hline \multicolumn{4}{|l|}{ Postprocedural MBG } \\
\hline MBG 0 & $0(0 \%)$ & $11(22 \%)$ & \multirow{4}{*}{0.001} \\
\hline MBG 1 & $3(6 \%)$ & $8(16 \%)$ & \\
\hline MBG 2 & $23(46 \%)$ & $17(34 \%)$ & \\
\hline MBG 3 & $24(48 \%)$ & $14(28 \%)$ & \\
\hline Corrected TIMI frame count, frames & $24.2 \pm 4.97$ & $31.28 \pm 6.7$ & $<0.0001$ \\
\hline
\end{tabular}

Continuous variables are expressed as mean and standard deviation whereas categorical variables are expressed as number (percentage). LAD means left anterior descending coronary artery; GP means glycoprotein; TIMI means thrombolysis in myocardial infarction; MBG means myocardial blush grade.

\subsection{In Hospital and Six-month Follow-up}

No in-hospital events occurred in both groups. There was no difference between both groups regarding peri-procedural and post-procedural bleeding complications. Two patients had minimal bleeding at the puncture site post-procedure and a third one had a small haematoma in the study group, while in the control group two patients had small haematomas. All hematomas were controlled and caused no short or long-term complications.

At 6-months follow-up, one patient in each group had a reinfarction, one patient in the study group had sustained a cerebrovascular stroke 3 months after the procedure, and one patient in the study group had a cardiac death 2 weeks after the procedure.

\section{Discussion}

Primary PCI with restoration of epicardial coronary blood flow is currently the gold standard therapy for eligible patients presenting with acute STEMI. It has dramatically improved survival and reduced MACE. The presence of heavy thrombus burden in such patients remains an obstacle towards the successful restoration of adequate coronary flow. No reflow, distal embolization and obstruction of the microvascular circulation are common causes. Managing heavy thrombus burden remains a challenge during primary PCI with several mechanical and pharmacological interventions proposed to improve outcome in such patients. Among the pharmacological tools available is the use of intracoronary thrombolytic therapy $[1,2,4,13,14]$. 
This study aimed to assess the effect of administration of $250,000 \mathrm{U}$ of intracoronary streptokinase during primary PCI in patients presenting with an acute anterior wall STEMI who had a definite thrombus in the LAD on both clinical and angiographic outcomes compared to conventional primary PCI.

The main findings of this study were that a significantly larger percentage of patients had post-procedural TIMI 3 flow grade, and MBG 2 or 3 in those treated with intracoronary streptokinase indicating better coronary perfusion. Corrected TIMI frame count was lower in the study group compared with those who didn't receive intracoronary thrombolytic therapy. Additionally, peak serum CK-MB level was significantly lower in the study group reflecting better reperfusion with less myocardial damage.

There was no difference between both groups regarding peri-procedural bleeding complications. No difference was found between both groups for the occurrence of MACE during a 6 month follow up period.

Sezer and his colleagues performed a study on 41 patients undergoing primary PCI. Patients randomly received either $250,000 \mathrm{U}$ of intracoronary streptokinase or no additional therapy after PCI. Coronary hemodynamic measurements were assessed during a coronary angiography performed two days later. Patients who received intracoronary streptokinase had significantly better measures of microvascular function (coronary flow reserve, microvascular resistance index, collateral flow index, mean coronary wedge pressure, systolic coronary wedge pressure, and diastolic deceleration time). Additionally, corrected TIMI frame count was lower in the streptokinase group. However, LV size and function showed no difference at 6 months follow up [13].

Another study performed by this group on 95 patients during primary PCI where 51 patients received 250,000 U of intracoronary streptokinase and 44 received no additional therapy examined the coronary hemodynamics after 2 days and reported better measures of microvascular function (coronary flow reserve, microvascular resistance index), lower corrected TIMI frame count, and better MBG in the streptokinase group. The authors concluded that the use of intracoronary streptokinase caused better microvascular perfusion. Additionally, at 6 months follow-up the streptokinase group showed a smaller infarct size on technetium-99m single-photon emission computed tomography imaging, higher LV ejection fraction and smaller LV volumes on echocardiography [14].

The randomized trial DISSOLUTION was performed to assess the effect of administration of intracoronary thrombolytic therapy (200,000 U of urokinase) to improve the efficacy of thrombus aspiration in patients undergoing primary PCI. 102 patients were randomized into two equal groups (urokinase versus normal saline). All patients had angiographic evidence of heavy thrombus burden in the culprit artery. An infusion microcatheter was used for delivery of urokinase, this was followed by manual thrombus aspiration. The authors reported higher post-procedural TIMI flow grade, higher MBG, and lower corrected TIMI frame count in the urokinase group. Additionally, they found that those in the urokinase group had lower MACE at 6-months flow-up. They concluded that local intra-thrombus administration of urokinase prior to manual thrombus aspiration improved post-procedural coronary flow, myocardial perfusion and 6-month clinical outcomes [15].

A meta-analysis was performed in 2015 comparing the benefits and risks of intracoronary thrombolytic therapy as an adjunct agent during primary PCI. It included three randomised studies on 131 patients, where 71 received intracoronary thrombolytic therapy and 60 received intracoronary placebo. The authors found that MBG was better in those receiving thrombolytic therapy compared to placebo with no difference in mortality. They concluded that using targeted intracoronary thrombolytic therapy is a safe and effective therapy to further improve the outcome of primary PCI [16].

Another study was performed using intracoronary prourokinase in 118 patients compared to normal saline in 112 patients using a balloon catheter during primary PCI. Patients in the pro-urokinase group had more complete ST-segment resolution, lower serum levels of CK-MB and troponin I, and better MBG. There was no difference regarding major or minor bleeding or MACE at 6-months follow-up. The authors concluded that intracoronary administration of prourokinase improved myocardial perfusion in STEMI patients [17].

Different studies examining the use of each of intracoronary tenecteplase [18] and alteplase [19] during primary PCI and found that both agents were safe for intracoronary administration with a trend towards improvement in thrombus burden, however, both agents were not found to improve outcome during primary PCI.

The current study does not mean to challenge the current standard procedures followed by interventional cardiologists during primary PCI. However, it shines a light on a slightly forgotten tool that is known to be effective ever since the inception of thrombolytic therapy and the ground-breaking research performed by Rentrop on intracoronary streptokinase in the 1980s. Intracoronary thrombolytic therapy may be helpful in specific patients where the interventional cardiologists expects microvacuolar injury and obstruction to occur such as in those with heavy thrombus burden during primary PCI [20-22].

Study limitations:

The limitations of the current study are that it was conducted on a relatively small number of patients at two medical centres. Use of intracoronary glycoprotein IIb/IIIa antagonists, although limited, was allowed at the operators' discretion, as well as, the use of balloon pre-dilatation prior to stenting.

\section{Conclusion}

The administration of intracoronary streptokinase during primary PCI in patients presenting with acute anterior STEMI with definite coronary thrombosis improves coronary 
perfusion by improving TIMI flow grade, MBG, and shortening corrected TIMI frame count without increasing the risk of major or minor bleeding. Its administration in such patients is an important tool that interventional cardiologists should consider in the setting of heavy thrombus burden during primary PCI.

\section{References}

[1] Ibanez B, James S, Agewall S et al. 2017 ESC Guidelines for the management of acute myocardial infarction in patients presenting with ST-segment elevation: The Task Force for the management of acute myocardial infarction in patients presenting with ST-segment elevation of the European Society of Cardiology (ESC). Eur Heart J. 2018 Jan 7; 39 (2): 119177.

[2] Levine GN, Bates ER, Blankenship JC et al. 2015 ACC/AHA/SCAI Focused Update on Primary Percutaneous Coronary Intervention for Patients With ST-Elevation Myocardial Infarction: An Update of the 2011 ACCF/AHA/SCAI Guideline for Percutaneous Coronary Intervention and the $2013 \mathrm{ACCF} / \mathrm{AHA}$ Guideline for the Management of ST-Elevation Myocardial Infarction: A Report of the American College of Cardiology/American Heart Association Task Force on Clinical Practice Guidelines and the Society for Cardiovascular Angiography and Interventions. Circulation. 2016 Mar 15; 133 (11): 1135-47.

[3] Mazhar J, Maschicharan M, Farshid A. Predictors and outcome of no-reflow post primary percutaneous coronary intervention for ST elevation myocardial infarction. IJC Heart Vasc. 2016; 10: 8-12.

[4] Rezkalla SH, Stankowski RV, Hanna J et al. Management of No-Reflow Phenomenon in the Catheterization Laboratory. JACC Cardiovasc Interv. 2017; 10 (3): 215-223.

[5] Gupta S, Gupta MM. No reflow phenomenon in percutaneous coronary interventions in ST-segment elevation myocardial infarction. Indian Heart J. 2016; 68 (4): 539-51.

[6] Boscarelli D, Vaquerizo B, Miranda-Guardiola F, et al. Intracoronary thrombolysis in patients with ST-segment elevation myocardial infarction presenting with massive intraluminal thrombus and failed aspiration. Eur Heart J 2014; 3: 229-36.

[7] Gibson CM, de Lemos JA, Murphy SA et al. Combination therapy with abciximab reduces angiographically evident thrombus in acute myocardial infarction: a TIMI 14 substudy. Circulation. 2001 May 29; 103 (21): 2550-4.

[8] Thygesen K, Alpert JS, Jaffe AS et al. Third universal definition of myocardial infarction. Circulation. 2012 Oct 16; 126 (16): 2020-35.

[9] Real Lebeau, Karim Serri, Maria Di Lorenzo et al.: Assessment of LVEF using a new 16-segment wall motion score in echocardiography. Echo Res Pract. 2018; 5 (2): 6369.

[10] Chesebro JH, Knatterud G, Roberts R et al. Thrombolysis in Myocardial Infarction (TIMI) Trial, Phase I: A comparison between intravenous tissue plasminogen activator and intravenous streptokinase. Clinical findings through hospital discharge. Circulation. 1987 Jul; 76 (1): 142-54.

[11] Van't Hof AW, Liem A, Suryapranata H et al. Angiographic assessment of myocardial reperfusion in patients treated with primary angioplasty for acute myocardial infarction: myocardial blush grade. Zwolle Myocardial Infarction Study Group. Circulation. 1998 Jun 16; 97 (23): 2302-6.

[12] Gibson CM, Cannon CP, Daley WL et al. TIMI frame count: a quantitative method of assessing coronary artery flow. Circulation. 1996 Mar 1; 93 (5): 879-88.

[13] Sezer M, Oflaz H, Gören T et al.: Intracoronary streptokinase after primary percutaneous coronary intervention. $\mathrm{N}$ Engl $\mathrm{J}$ Med. 2007; 356 (18): 1823-34.

[14] Sezer M, Çimen A, Aslanger E et al.: Effect of intracoronary streptokinase administered immediately after primary percutaneous coronary intervention on long-term left ventricular infarct size, volumes, and function. J Am Coll Cardiol 2009; 54: 1065-1071.

[15] Greco C, Pelliccia F, Tanzilli G et al. Usefulness of local delivery of thrombolytics before thrombectomy in patients with ST-segment elevation myocardial infarction undergoing primary percutaneous coronary intervention (the delivery of thrombolytics before thrombectomy in patients with STsegment elevation myocardial infarction undergoing primary percutaneous coronary intervention [DISSOLUTION] randomized trial). Am J Cardiol. 2013; 112 (5): 630-635.

[16] Bainey KR, Welsh RC, Mehta S. Meta-analysis of intracoronary thrombolytic as an adjunct to primary percutaneous coronary intervention in ST-segment elevation myocardial infarction. Canadian Journal of Cardiology 2015; 31 (10): S1-313.

[17] Geng W, Zhang Q, Liu J, et al. A randomized study of prourokinase during primary percutaneous coronary intervention in acute ST-segment elevation myocardial infarction. J Interv Cardiol. 2018 Apr; 31 (2): 136-143.

[18] Gibson CM, Kumar V, Gopalakrishnan L, et al. Feasibility and Safety of Low-Dose Intra-Coronary Tenecteplase During Primary Percutaneous Coronary Intervention for ST-Elevation Myocardial Infarction (ICE T-TIMI 49). Am J Cardiol. 2020 Feb 15; 125 (4): 485-490.

[19] McCartney PJ, Eteiba H, Maznyczka AM, et al. Effect of Low-Dose Intracoronary Alteplase During Primary Percutaneous Coronary Intervention on Microvascular Obstruction in Patients With Acute Myocardial Infarction: A Randomized Clinical Trial. JAMA. 2019 Jan 1; 321 (1): 5668 .

[20] Rentrop, P. et al. Selective intracoronary thrombolysis in acute myocardial infarction and unstable angina pectoris. Circulation 1981; 63, 307-317.

[21] Jayagopal PB, Sarjun Basha KM. Intracoronary tenecteplase in STEMI with massive thrombus. Indian Heart J. 2018 May Jun; 70 (3): 446-449.

[22] Agarwal SK, Agarwal S. Role of Intracoronary Fibrinolytic Therapy in Contemporary PCI Practice. Cardiovasc Revasc Med. 2019 Dec; 20 (12): 1165-1171. 